\title{
Effects of Variable Viscosity and Thermal Conductivity on the MHD Flow of Micropolar Fluid on a Continuous Moving Surface
}

\author{
Surajit Dutta \\ Department of Mathematics \\ C. N. B. College, Bokakhat \\ Assam, India
}

\author{
G. C. Hazarika \\ Department of Mathematics \\ Dibrugarh University, Dibrugarh \\ Assam, Indiia
}

\begin{abstract}
The effects of variable viscosity and thermal conductivity on the MHD flow of micropolar fluid on a continuous moving surface in the presence of a transverse magnetic field are studied considering the viscosity and thermal conductivity as the inverse linear functions of temperature where the microinertia density is assumed to be variable and not constant, as many other earlier published papers. Also, the viscous dissipation effect is taken into account. The partial differential equations governing the flow and heat transfer of the problem are transformed into dimensionless form of ordinary differential equations by using similarity substitutions. The governing boundary value problems so obtained are then solved numerically using Runge-Kutta method. The effects of various parameters viz. viscosity parameter, thermal conductivity parameter, magnetic parameter, coupling constant parameter, Eckert number and Prandtl number on velocity, micro-rotation, temperature field and micro-inertia density are studied and results are presented graphically. The co-efficient of skin-friction and Nusselt number are also computed and presented in tabular form.
\end{abstract}

\section{Keywords}

Micropolar fluid, variable viscosity and thermal conductivity, MHD Flow

\section{INTRODUCTION}

The concept of micropolar fluid derives from the need to model the flow of fluids that contain rotating microconstituents. In micropolar fluid theories, each particle has a finite size and constitutes a micro structure, which can rotate about the centre of the volume element described by the micro-rotation vector. This local rotation of the particles is in addition to the usual rigid body motion of the entire volume element. In micropolar fluid theory, the laws of classical mechanics are augmented with additional equations that account for conservation of micro-inertia moments and balance of first stress moments that arise due to consideration of the microstructure in a material.

The effects of magneto hydrodynamics on micropolar fluid flow has become important due to several engineering applications such as in MHD generators, designing cooling system for nuclear reactor, flow meters etc., where the micro concentration provides an important parameter for deciding the rate of heat flow. By simulating it one can obtain the desired temperature in such equipments. Several investigations have made theoretical and experimental studies of micropolar fluid flow in the presence of a transverse magnetic field during the last few decades. Assuming fluid viscosity as a linear function of temperature the effect of variable viscosity on MHD natural convection in micropolar fluids was studied by M. Abd El-Hakiem et al. [1]. Gorla et al. [7] investigated the Magneto hydrodynamic free convection boundary layer flow of a thermo micropolar fluid over a vertical plate. Gorla [5] studied the flow of a micropolar fluid over a flat plate. Ishak et al. [10] studied the problem of steady boundary layer flow and heat transfer of a micropolar fluid on an isothermal continuously moving plane surface assuming that the micro-inertia density is variable and not constant. Muthucumaraswami et al. [13] investigated the magnetic field effects on flow past an accelerated isothermal vertical plate with heat and mass diffusion. Modather M. et al. [14] studied variable viscosity effect on heat transfer over a continuous moving surface with variable internal heat generation in micropolar fluids. Peddieson et al. [15] investigated boundary layer theory for micro polar fluid. Rajesh [16] investigated the MHD free convection flow past an accelerated vertical porous plate with variable temperature through a porous medium. Salem A.M. et al. [17] studied influence of variable viscosity and thermal conductivity on flow of micropolar fluid past a continuously moving plate with suction or injection. Sarma and Hazarika [18] studied the effects of variable viscosity and thermal conductivity on heat and mass transfer flow of Newtonian fluid along a vertical plate in the presence of magnetic field. They [19] investigated effects of variable viscosity and thermal conductivity on combined free-forced convection and mass transfer flow passed a vertical porous plate. Hoyt J. W. et al. [9] and Vogel W. M. et al. [21] conducted experiments with fluids containing small amount of polymeric additives.

Soundalgekar V. M. et al. [20] solved the problem of steady boundary layer flow and heat transfer of a micropolar fluid due to a continuously moving surface by considering that the microinertia density is constant. Ishak A. et al. [10] studied the flow of a micropolar fluid on a continuous moving surface.

The main objective of our present work is to extend the work of Ishak A. et al. [10] to study the effects of variable viscosity and thermal conductivity on the flow of MHD micropolar fluid on a continuous moving surface in presence of a transverse magnetic field. Viscosity and thermal conductivity are assumed to be inverse linear functions of temperature. The governing partial differential equations are reduced in to ordinary differential equations by similarity transformations. The problem is then solved numerically using Runge-kutta shooting algorithm with iteration process.

\section{MATHEMATICAL FORMULATION OF THE PROBLEM}

Let us consider the flow of an incompressible micropolar fluid medium at rest in presence of a transverse magnetic field, where a flat plate emerges from the slot of an extrusion die at a constant velocity $U_{w}$ and continuously moves in to the fluid. 
The temperature of the plate is assumed as $T_{w}$ and that of the ambient fluid is $T_{\infty}$, where we assume that $T_{w}>T_{\infty}$ (the plate is heated). The origin of the Cartesian coordinate system is placed at the location where the plate is drawn into the fluid medium with the $x$-axis measured along the plate in right direction and the $y$-axis is taken normal to it. Also a magnetic field of constant intensity is assumed to be applied normal to the vertical plate and the electrical conductivity of the fluid is assumed to be so small that the induced magnetic field can be neglected in comparison to the applied magnetic field. The applied magnetic field is primary in the $y$-direction and is a function of $x$ only. $(u(y), 0)$ is the velocity component and $N$ is the component of micro rotation perpendicular to the $x y$-plane.Under these assumptions the governing equations of the problem are:

\subsection{Basic Equations:}

\section{Equation of continuity:}

$$
\frac{\partial u}{\partial x}+\frac{\partial v}{\partial y}=0
$$

\section{Momentum equation:}

$$
u \frac{\partial u}{\partial x}+v \frac{\partial u}{\partial y}=\frac{\partial}{\partial y}\left(v \frac{\partial u}{\partial y}\right)+\frac{\kappa}{\rho}\left(\frac{\partial N}{\partial y}+\frac{\partial^{2} u}{\partial y^{2}}\right)-\frac{\sigma B^{2}}{\rho} u
$$

\section{Angular momentum equation:}

$$
\frac{\partial}{\partial y}\left(\gamma \frac{\partial N}{\partial y}\right)-\kappa\left(2 N+\frac{\partial u}{\partial y}\right)
$$

Equation for microinertia density:

$$
u \frac{\partial j}{\partial x}+v \frac{\partial j}{\partial y}=0
$$

\section{Energy equation:}

$$
u \frac{\partial T}{\partial x}+v \frac{\partial T}{\partial y}=\frac{1}{\rho c_{\mathrm{p}}} \frac{\partial}{\partial y}\left(\lambda \frac{\partial T}{\partial y}\right)+\frac{1}{\rho c_{\mathrm{p}}}(\mu+\kappa)\left(\frac{\partial u}{\partial y}\right)^{2}
$$

The equation of continuity is being identically satisfied by velocity profile $(u(y), 0)$. It should be mentioned that $N=0$ indicates strong concentration by Gurum and Smith [8] and represents concentrated particles flows in which the microelements close to the wall surface are unable to translate or rotate. It is known (Gorla [6]) that $N$ is the total spin of microstructure and fluid media in the flow field. In some cases, the microstructure effects become negligible and the fluid behaves like an ordinary (Newtonian) viscous flow. Therefore, if we state that $N=$ angular velocity is a valid solution, then this is possible only if

$$
\gamma=\left(\mu_{\infty}+\frac{\kappa}{2}\right) j=\mu_{\infty}\left(1+\frac{K}{2}\right) j, \quad \text { where } \mathrm{K}=\frac{\kappa}{v_{\infty} \rho},
$$

coupling constant parameter.

Following Lai and Kulacki [9] we assume the viscosity as inverse linear functions of temperature, i.e.

$$
\begin{aligned}
\frac{1}{\mu}= & \frac{1}{\mu_{\infty}}\left[1+\delta\left(T-T_{\infty}\right)\right], \text { or } \frac{1}{\mu}=a\left(T-T_{r}\right) \\
& \text { where } a=\frac{\delta}{\mu_{\infty}} \text { and } T_{r}=T_{\infty}-\frac{1}{\delta}
\end{aligned}
$$

Following Khound and Hazarika[11] we assume the thermal conductivity as inverse linear functions of temperature, i.e.

$$
\frac{1}{\lambda}=\frac{1}{\lambda_{\infty}}\left[1+\xi\left(T-T_{\infty}\right)\right], \text { or } \frac{1}{\lambda}=b\left(T-T_{c}\right)
$$

where $b=\frac{\xi}{\lambda_{\infty}}, T_{c}=T_{\infty}-\frac{1}{\xi}$ and $a, b, T_{r}$ and $T_{c}$ are constants and their values depend on the reference state and thermal properties of the fluid i.e. $v$ and $\lambda$.

The appropriate boundary conditions are

$$
\left.\begin{array}{ll}
v=j=0, u=U_{w}, N=-n \frac{\partial u}{\partial y}, T=T_{w}, & \text { at } \mathrm{y}=0 \\
u \rightarrow 0, T \rightarrow T_{\infty}, \quad N \rightarrow 0 & \text { as } \mathrm{y} \rightarrow \infty
\end{array}\right\} . .
$$

We introduce the following non-dimensional variables

$$
\left.\begin{array}{c}
\psi=\left(2 v_{\infty} U_{w} x\right)^{\frac{1}{2}} f(\eta), \quad \eta=\left(\frac{U_{w}}{2 v_{\infty} x}\right)^{\frac{1}{2}} y, \frac{T-T_{\infty}}{T_{w}-T_{\infty}}=\theta(\eta), \\
N=\left(\frac{U_{w}^{3}}{2 v_{\infty} x}\right)^{\frac{1}{2}} h(\eta), \\
j=\frac{2 v_{\infty} x}{U_{w}} g(\eta), \quad \gamma=\left(\mu+\frac{k}{2}\right) \frac{2 v_{\infty} x}{U_{w}} g(\eta)
\end{array}\right\} \ldots
$$

where $\psi$ is the stream function defined in the usual way as $u=\frac{\partial \psi}{\partial y}$ and $v=-\frac{\partial \psi}{\partial x}$.

Substituting above transformations in equations (2) - (5), we get

$$
\begin{aligned}
& \left(\frac{\theta_{r}}{\theta-\theta_{r}}-K\right) f^{\prime \prime \prime}=\frac{\theta_{r}}{\left(\theta-\theta_{r}\right)^{2}} f^{\prime \prime} \theta^{\prime}+f f^{\prime \prime}+K h^{\prime}-M f^{\prime} . . \\
& \left(1+\frac{1}{2} K\right)\left(g h^{\prime}\right)^{\prime}-K\left(2 h+f^{\prime \prime}\right)=0 . \\
& 2 f^{\prime} g-f g^{\prime}=0 \\
& \theta^{\prime \prime}=P_{r} \frac{\theta-\theta_{c}}{\theta_{c}} f \theta^{\prime}+\frac{\theta^{\prime 2}}{\theta-\theta_{c}}+E_{c} \frac{\theta-\theta_{c}}{\theta_{c}}\left[K-\frac{\theta_{r}}{\theta-\theta_{r}}\right]\left(f^{\prime \prime}\right)^{2} \ldots
\end{aligned}
$$

and the boundary conditions (9)become

$$
\left.\begin{array}{rl}
\eta=0: & f=0, f^{\prime}=1, g=0, \theta=1, h=-n f^{\prime \prime} \\
\eta & \rightarrow \infty: f^{\prime}=0, \theta=0, h=0
\end{array}\right\}
$$

where $P_{r}$ is the Prandtl number and $E_{c}$ is the Eckert number, which is defined as

$$
E_{c}=\frac{U_{w}^{2}}{c_{p}\left(T_{w}-T_{\infty}\right)}
$$

The solution of equation (12) is

$$
g=c f^{2}
$$

where $c$ is a dimensionless arbitrary constant of integration.

Using equation (16) we have from equation (11)

$$
\left(1+\frac{1}{2} K\right)\left(2 c f f^{\prime} h^{\prime}+c f^{2} h^{\prime \prime}\right)-K\left(2 h+f^{\prime \prime}\right)=0
$$

The physical quantities of interest in this problem are the skin friction coefficient $c_{f}$, local Nusselt number $N u_{x}$ which indicate physically wall shear stress and rate of heat transfer respectively. For micropolar boundary layer flow, the wall shear stress $\tau_{w}$ is given by

$$
\tau_{w}=\left[(\mu+\kappa) \frac{\partial u}{\partial y}+\kappa N\right]_{y=0}
$$


The skin friction coefficient $c_{f}$ can be defined as

$$
c_{f}=\frac{2 \tau_{w}}{\rho U_{w}^{2}}=\frac{\sqrt{ } 2\left\{\frac{\theta_{r}}{\theta_{r}-1}+(1-n) K\right\}}{\left(R e_{x}\right)^{1 / 2}} f^{\prime \prime}(0)
$$

The rate of heat transfer from the plate is given by

$$
q_{w}=-\lambda\left[\frac{\partial T}{\partial y}\right]_{y=0}=\lambda_{\infty} \frac{\theta_{c}}{1-\theta_{c}}\left(T_{w}-T_{\infty}\right)\left(\frac{U_{w}}{2 v_{\infty} x}\right)^{1 / 2} \theta^{\prime}(0)
$$

$$
N u_{x}=\frac{1}{\sqrt{2}} \frac{\theta_{c}}{1-\theta_{c}}\left(R e_{x}\right)^{1 / 2} \theta^{\prime}(0)
$$

where $R e_{x}=U_{w} x / v_{\infty}$ is the local Reynolds number.

\section{RESULTS AND DISCUSSION}

The nonlinear ordinary differential equations (10), (13) and (17), together with the boundary conditions (14) are solved for various combination of the parameters involved in the equations using an algorithm based on the shooting method and presented results for the dimensionless velocity distribution, micro-rotation distribution, temperature distribution and micro-inertia density with the variation of different parameters.

The numerical values of different parameters are taken as $M=1, P r=0.7, \theta_{c}=-14, \theta_{r}=-12, K=1, N=0.5$, $E_{c}=0.05$ unless otherwise stated. The variations in velocity distribution, temperature distribution, angular velocity distribution, micro inertia density distribution are illustrated in figures (1) - (17) with the variation of different parameters.

Fig. (1) - (4) represent the variations in dimensionless velocity distribution with magnetic parameter $M$, viscosity parameter $\theta_{r}$, coupling constant parameter $K$ and micro rotation parameter $N$. From fig. (1) it is clear that velocity decreases with increase of magnetic parameter $M$. It is due to the fact that the application of transverse magnetic field will result a resistive force (Lorentz force) similar to drag force, which tends to resist the fluid flow and thus reducing its velocity. From fig. (2) it is clear that velocity decreases for increase of viscosity parameter $\theta_{r}$. From fig. (3) we observe that velocity increases for increase of coupling constant parameter $K$. It is because when $K$ increases viscosity will decrease and as a result velocity will increase. Fig. (4) shows that velocity will decrease for increase of micro rotation parameter $N$.

Figures (5) - (9) display the variations of dimensionless temperature profile $\theta(\eta)$ with the variation of magnetic parameter $M$, viscosity parameter $\theta_{r}$, thermal conductivity parameter $\theta_{c}$, coupling constant parameter $K$, and micro rotation parameter $N$. Figure (5) and (6) shows that temperature increase with increase of magnetic parameter $M$ and viscosity parameter $\theta_{r}$ respectively. As in both cases a resistive force will act against the fluid flow and as a result temperature will increase. In figure (7) also temperature increases for increase of thermal conductivity parameter $\theta_{c}$. But temperature decrease [fig.(8)] for increase of coupling constant parameter $K$. Also in figure (9) it shows that temperature increases for increase of micro rotation parameter $N$.

Figure (10) - (13) shows the angular velocity distribution for various values of magnetic parameter $M$, viscosity parameter $\theta_{r}$, coupling constant parameter $K$ and micro rotation parameter $N$. Figure (10) shows that angular velocity increases for the increase of magnetic parameter $M$. Figure (11) shows that angular velocity will increase for increase of viscosity parameter $\theta_{r}$. Figure (12) shows that angular velocity decreases for increase of coupling constant parameter $K$ while figure (13) shows that angular velocity will increase for increase of micro rotation parameter $N$.

Figure (14) - (15) shows that the micro inertia density profile for various values of $M$ and $\theta_{r}$. Figure (14) shows that microinertia density will decrease for the increase of magnetic parameter $M$ and figure (15) also shows that microinertia density will decrease for the increase of viscosity parameter $\theta_{r}$.

\section{FIGURES}

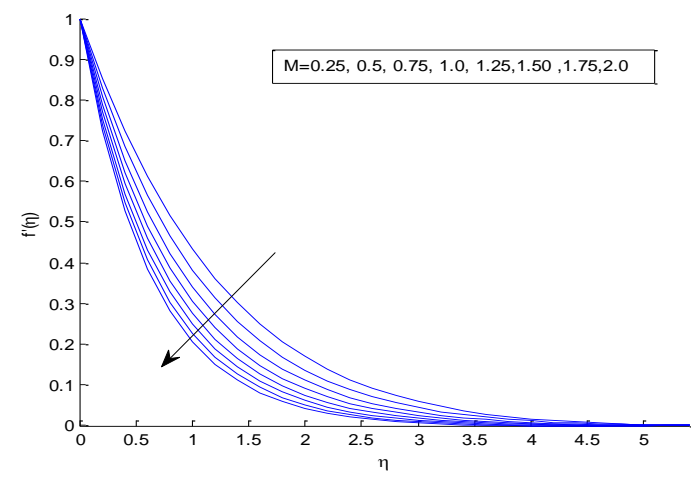

Fig.1. Velocity profiles for various $\mathrm{M}(\mathrm{Pr}=0.7)$

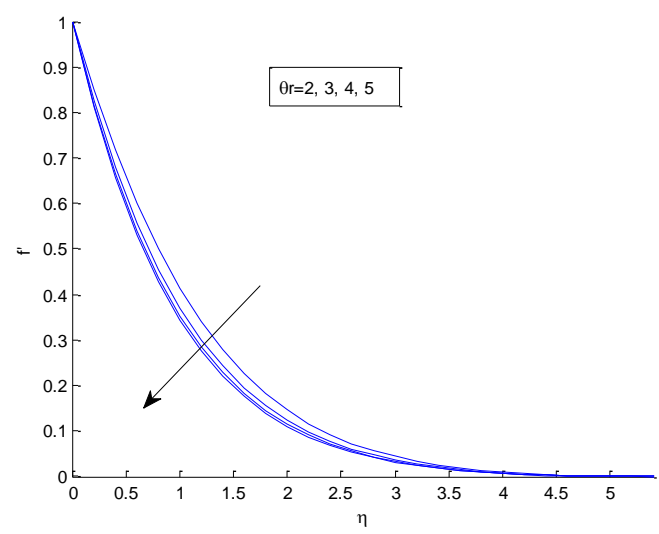

Fig.2. Velocity profiles for various $\theta_{r}(\operatorname{Pr}=0.7)$

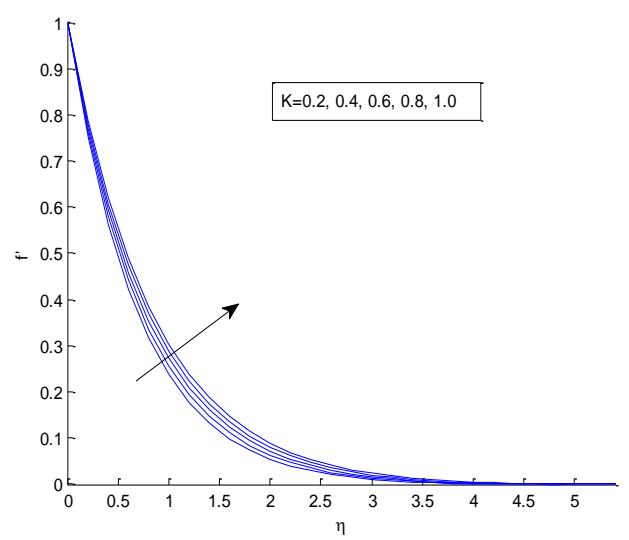

Fig.3. Velocity profiles for various $\mathrm{K}(\mathrm{Pr}=0.7$ 


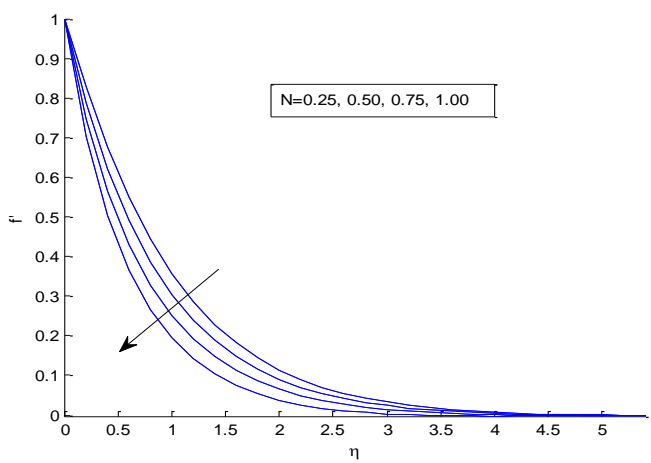

Fig.4. Velocity profiles for various $\mathrm{N}(\operatorname{Pr}=0.7)$

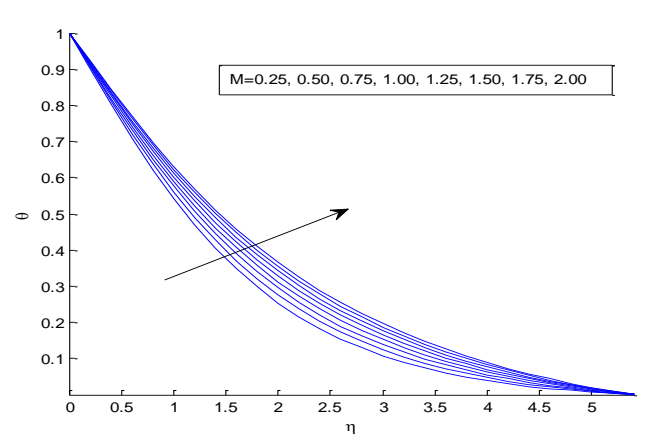

Fig.5. Temperature profiles for various $M(P r=0.7)$

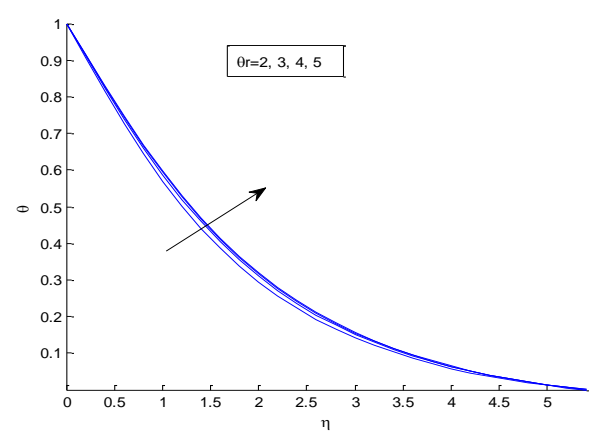

Fig.6. Temperature profiles for various $\theta_{r}(\operatorname{Pr}=0.7)$

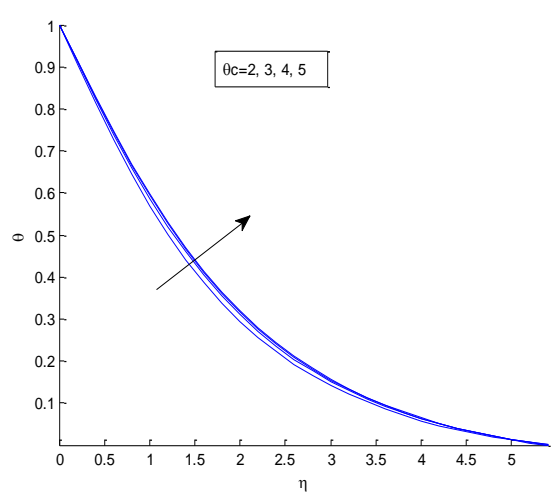

Fig.7. Temperature profiles for various $\theta_{c}(\operatorname{Pr}=0.7)$

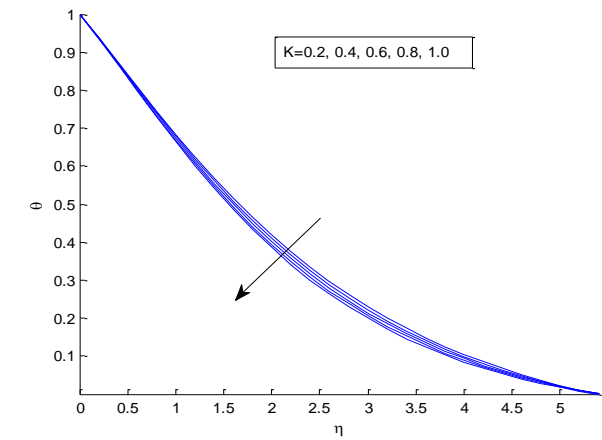

Fig.8.Temperature profiles for various $\mathrm{K}(\mathrm{Pr}=0.7)$

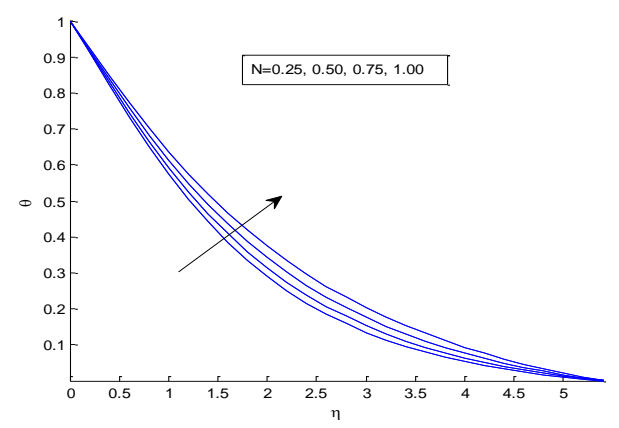

Fig.9. Temperature profiles for various $\mathrm{N}(\operatorname{Pr}=0.7)$

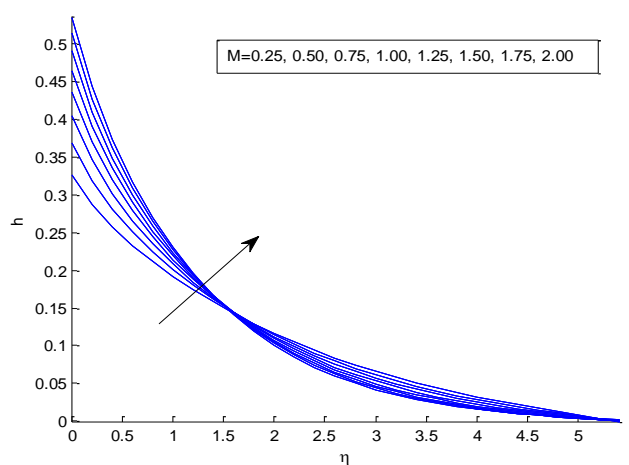

Fig.10. Angular velocity profile for various M (Pr=0.7)

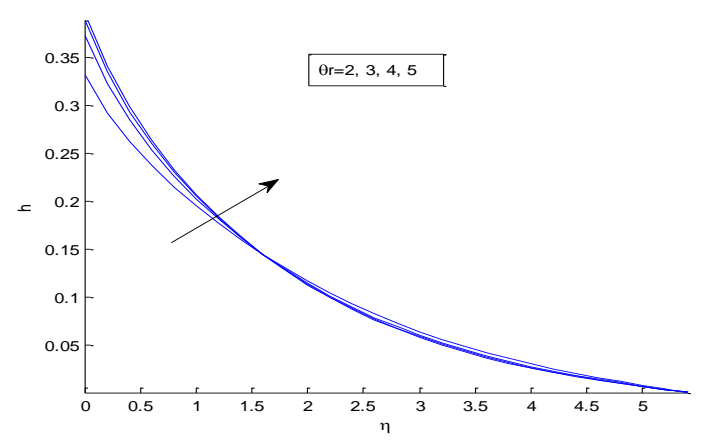

Fig.11. Angular velocity profiles for various $\theta_{r}(\operatorname{Pr}=0.7)$ 


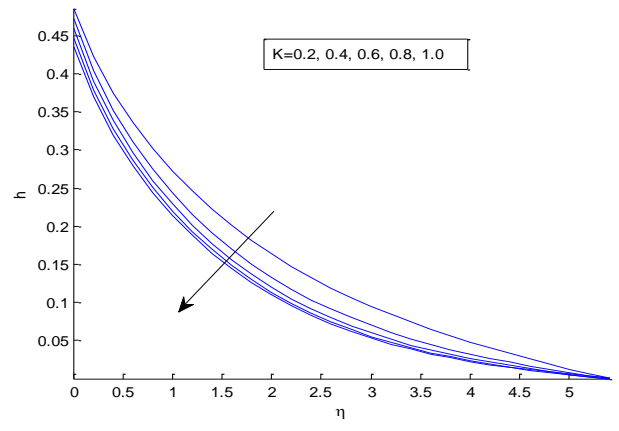

Fig.12. Angular velocity profiles for various $\mathrm{K}(\operatorname{Pr}=0.7)$

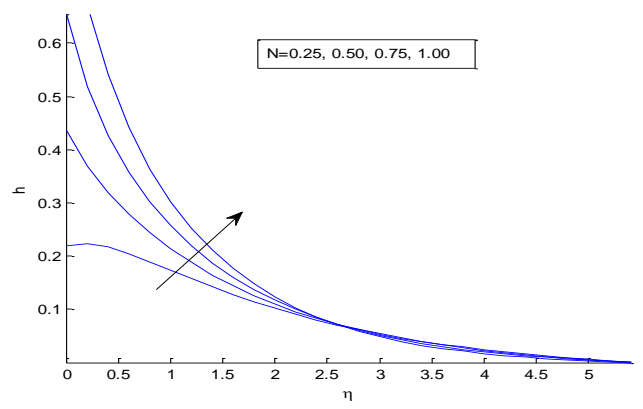

Fig.13. Angular velocity profiles for various $\mathrm{N}(\operatorname{Pr}=0.7)$

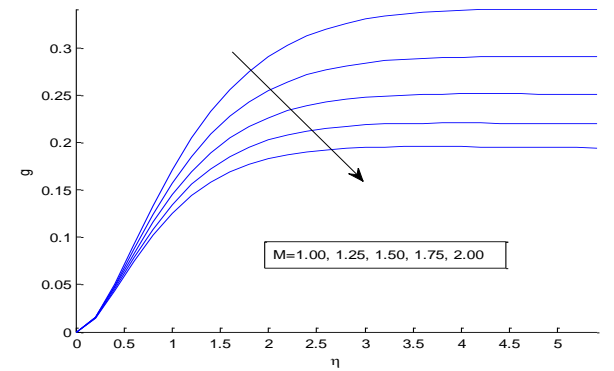

Fig.14. Micro inertia density profile for various $M$ $(\operatorname{Pr}=0.7)$

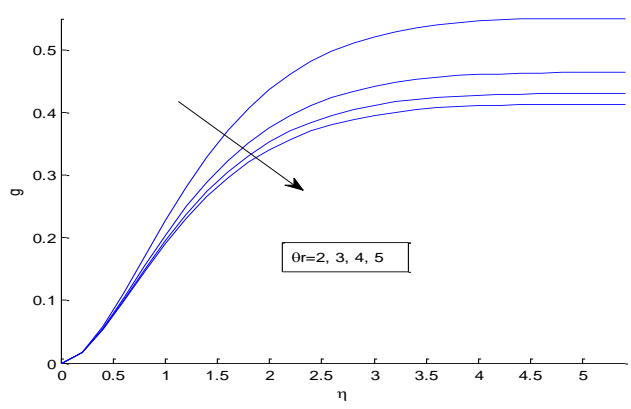

Fig.15. Micro inertia density profiles for various $\theta_{r}$ (Pr=0.7)

\section{TABLES}

Table I: Missing values and physical quantities with the variation of $\theta \mathrm{c}$ and $\mathrm{K}$

\begin{tabular}{|c|c|c|c|c|c|c|c|c|c|c|c|}
\hline$\theta \mathrm{c}$ & \multicolumn{5}{|l|}{$\rightarrow$} & \multicolumn{6}{|c|}{3} \\
\hline K & $f^{\prime \prime}(0)$ & $\theta^{\prime}(0)$ & $h^{\prime}(0)$ & $c_{f}$ & $N u_{x}$ & $\mathrm{~K}$ & $f^{\prime \prime}(0)$ & $\theta^{\prime}(0)$ & $h^{\prime}(0)$ & $c_{f}$ & $N u_{x}$ \\
\hline 0.2 & -0.69267 & -0.46454 & -0.23404 & -1.00219 & 0.65696 & 0.2 & -0.79785 & -0.44587 & -0.28879 & -1.15436 & 0.472912 \\
\hline 0.4 & -0.68451 & -0.46609 & -0.23777 & -1.08718 & 0.659143 & 0.4 & -0.78485 & -0.44816 & -0.30372 & -1.24656 & 0.47534 \\
\hline 0.6 & -0.67546 & -0.46767 & -0.23425 & -1.16833 & 0.661385 & 0.6 & -0.77084 & -0.45051 & -0.3039 & -1.33331 & 0.477842 \\
\hline 0.8 & -0.66638 & -0.46915 & -0.22891 & -1.24688 & 0.663482 & 0.8 & -0.75702 & -0.45275 & -0.29923 & -1.41647 & 0.480217 \\
\hline 1 & -0.65753 & -0.4705 & -0.2232 & -1.32331 & 0.665387 & 1 & -0.74374 & -0.45482 & -0.29274 & -1.4968 & 0.48241 \\
\hline$\theta c$ & \multicolumn{5}{|c|}{4} & \multicolumn{6}{|c|}{5} \\
\hline K & $f^{\prime \prime}(0)$ & $\theta^{\prime}(0)$ & $h^{\prime}(0)$ & $c_{f}$ & $N u_{x}$ & K & $\mathrm{f}^{\prime \prime}(0)$ & $\theta^{\prime}(0)$ & $h^{\prime}(0)$ & $c_{f}$ & $N u_{x}$ \\
\hline 0.2 & -0.84087 & -0.43777 & -0.31047 & -1.21662 & 0.412736 & 0.2 & -0.86435 & -0.43326 & -0.32213 & -1.25058 & 0.382951 \\
\hline 0.4 & -0.82524 & -0.44049 & -0.32968 & -1.3107 & 0.415295 & 0.4 & -0.84708 & -0.43624 & -0.34358 & -1.34539 & 0.385581 \\
\hline 0.6 & -0.80873 & -0.44325 & -0.33105 & -1.39886 & 0.417901 & 0.6 & -0.82909 & -0.43925 & -0.34551 & -1.43407 & 0.388241 \\
\hline 0.8 & -0.79264 & -0.44587 & -0.32637 & -1.48312 & 0.420372 & 0.8 & -0.81167 & -0.44209 & -0.34075 & -1.51873 & 0.390758 \\
\hline 1 & -0.7773 & -0.4483 & -0.31933 & -1.56434 & 0.422656 & 1 & -0.79514 & -0.44472 & -0.33335 & -1.60025 & 0.393083 \\
\hline
\end{tabular}


Table II: Missing values and physical quantities with variation of $\theta \mathrm{c}$ and $\mathrm{N}$

\begin{tabular}{|c|c|c|c|c|c|c|c|c|c|c|c|}
\hline$\theta c$ & $\rightarrow$ & & 2 & & & \multicolumn{6}{|c|}{3} \\
\hline$N$ & $f^{\prime \prime}(0)$ & $\theta^{\prime}(0)$ & $h^{\prime}(0)$ & $c_{f}$ & $N u_{x}$ & $N$ & $f^{\prime \prime}(0)$ & $\theta^{\prime}(0)$ & $h^{\prime}(0)$ & $c_{f}$ & $N u_{x}$ \\
\hline 0.25 & -0.74911 & -0.46918 & 0.113354 & -1.77246 & 0.497638 & 0.25 & -0.74911 & -0.46918 & 0.113354 & -1.77246 & 0.497638 \\
\hline 0.5 & -0.74374 & -0.45482 & -0.29274 & -1.4968 & 0.48241 & 0.5 & -0.74374 & -0.45482 & -0.29274 & -1.4968 & 0.48241 \\
\hline 0.75 & -0.74402 & -0.43861 & -0.69055 & -1.23431 & 0.465217 & 0.75 & -0.74402 & -0.43861 & -0.69055 & -1.23431 & 0.465217 \\
\hline 1 & -0.74893 & -0.42024 & -1.08745 & -0.97767 & 0.445729 & 1 & -0.74893 & -0.42024 & -1.08745 & -0.97767 & 0.445729 \\
\hline$\theta c$ & \multicolumn{5}{|c|}{4} & \multicolumn{6}{|c|}{5} \\
\hline $\mathrm{N}$ & $f^{\prime \prime}(0)$ & $\theta^{\prime}(0)$ & $h^{\prime}(0)$ & $c_{f}$ & $N u_{x}$ & $\mathrm{~N}$ & $f^{\prime \prime}(0)$ & $\theta^{\prime}(0)$ & $h^{\prime}(0)$ & $c_{f}$ & $N u_{x}$ \\
\hline 0.25 & -0.78305 & -0.46399 & 0.104238 & -1.85276 & 0.437457 & 0.25 & -0.80106 & -0.46117 & 0.099484 & -1.89538 & 0.407617 \\
\hline 0.5 & -0.7773 & -0.4483 & -0.31933 & -1.56434 & 0.422656 & 0.5 & -0.79514 & -0.44472 & -0.33335 & -1.60025 & 0.393083 \\
\hline 0.75 & -0.77805 & -0.43049 & -0.73415 & -1.29076 & 0.405869 & 0.75 & -0.79622 & -0.42602 & -0.75725 & -1.32091 & 0.376554 \\
\hline 1 & -0.7841 & -0.41018 & -1.14854 & -1.02359 & 0.386721 & 1 & -0.80298 & -0.40462 & -1.18104 & -1.04823 & 0.357635 \\
\hline
\end{tabular}

Table III: Missing values and physical quantities with the variation of $\theta \mathrm{r}$ and $\mathrm{K}$

\begin{tabular}{|c|c|c|c|c|c|c|c|c|c|c|c|}
\hline$\theta \mathrm{r}$ & $\longrightarrow$ & & 2 & & & \multicolumn{6}{|c|}{3} \\
\hline $\mathrm{K}$ & $f^{\prime \prime}(0)$ & $\theta^{\prime}(0)$ & $h^{\prime}(0)$ & $c_{f}$ & $N u_{x}$ & K & $f^{\prime \prime}(0)$ & $\theta^{\prime}(0)$ & $h^{\prime}(0)$ & $c_{f}$ & $N u_{x}$ \\
\hline 0.2 & -0.9678 & -0.22627 & -0.37192 & -2.87422 & 0.149329 & 0.2 & -0.9678 & -0.22627 & -0.37192 & -2.87422 & 0.149329 \\
\hline 0.4 & -0.94137 & -0.22895 & -0.40218 & -2.92886 & 0.151099 & 0.4 & -0.94137 & -0.22895 & -0.40218 & -2.92886 & 0.151099 \\
\hline 0.6 & -0.91566 & -0.23161 & -0.40567 & -2.97836 & 0.152851 & 0.6 & -0.91566 & -0.23161 & -0.40567 & -2.97836 & 0.152851 \\
\hline 0.8 & -0.89164 & -0.23414 & -0.39987 & -3.02631 & 0.154524 & 0.8 & -0.89164 & -0.23414 & -0.39987 & -3.02631 & 0.154524 \\
\hline 1 & -0.86939 & -0.23652 & -0.39039 & -3.07375 & 0.156096 & 1 & -0.86939 & -0.23652 & -0.39039 & -3.07375 & 0.156096 \\
\hline$\theta \mathrm{r}$ & \multicolumn{5}{|c|}{4} & \multicolumn{6}{|c|}{5} \\
\hline $\mathrm{K}$ & $f^{\prime \prime}(0)$ & $\theta^{\prime}(0)$ & $h^{\prime}(0)$ & $c_{f}$ & $N u_{x}$ & K & $f^{\prime \prime}(0)$ & $\theta^{\prime}(0)$ & $h^{\prime}(0)$ & $c_{f}$ & $N u_{x}$ \\
\hline 0.2 & -0.96844 & -0.31068 & -0.37236 & -1.96308 & 0.205038 & 0.2 & -0.96856 & -0.32663 & -0.37245 & -1.84916 & 0.215562 \\
\hline 0.4 & -0.94198 & -0.31427 & -0.40272 & -2.04264 & 0.207407 & 0.4 & -0.94209 & -0.33036 & -0.40282 & -1.93185 & 0.218029 \\
\hline 0.6 & -0.91623 & -0.31779 & -0.40625 & -2.11639 & 0.209728 & 0.6 & -0.91633 & -0.33402 & -0.40636 & -2.00863 & 0.220443 \\
\hline 0.8 & -0.89218 & -0.3211 & -0.40047 & -2.18699 & 0.211918 & 0.8 & -0.89227 & -0.33747 & -0.40057 & -2.08207 & 0.222716 \\
\hline 1 & -0.8699 & -0.32419 & -0.39098 & -2.2554 & 0.213952 & 1 & -0.86999 & -0.34066 & -0.39108 & -2.15311 & 0.224823 \\
\hline
\end{tabular}


Table IV: Missing values and physical quantities with the variation of $\theta \mathrm{r}$ and $\mathrm{N}$

\begin{tabular}{|c|c|c|c|c|c|c|c|c|c|c|c|}
\hline$\theta_{r}$ & \multicolumn{5}{|c|}{2} & \multicolumn{6}{|c|}{3} \\
\hline $\mathrm{N}$ & $f^{\prime \prime}(0)$ & $\theta^{\prime}(0)$ & $h^{\prime}(0)$ & $c_{f}$ & $N u_{x}$ & $\mathrm{~N}$ & $f^{\prime \prime}(0)$ & $\theta^{\prime}(0)$ & $h^{\prime}(0)$ & $c_{f}$ & $N u_{x}$ \\
\hline 0.25 & -0.87538 & -0.24811 & 0.081024 & -3.40441 & 0.163743 & 0.25 & -0.87538 & -0.24811 & 0.081024 & -3.40441 & 0.163743 \\
\hline 0.5 & -0.86939 & -0.23652 & -0.39039 & -3.07375 & 0.156096 & 0.5 & -0.86939 & -0.23652 & -0.39039 & -3.07375 & 0.156096 \\
\hline 0.75 & -0.87269 & -0.22377 & -0.85269 & -2.77689 & 0.147679 & 0.75 & -0.87269 & -0.22377 & -0.85269 & -2.77689 & 0.147679 \\
\hline 1 & -0.88354 & -0.20956 & -1.31707 & -2.49901 & 0.1383 & 1 & -0.88354 & -0.20956 & -1.31707 & -2.49901 & 0.1383 \\
\hline$\theta_{r}$ & \multicolumn{5}{|c|}{4} & \multicolumn{6}{|c|}{5} \\
\hline $\mathrm{N}$ & $f^{\prime \prime}(0)$ & $\theta^{\prime}(0)$ & $h^{\prime}(0)$ & $c_{f}$ & $N u_{x}$ & $\mathrm{~K}$ & $f^{\prime \prime}(0)$ & $\theta^{\prime}(0)$ & $h^{\prime}(0)$ & $c_{f}$ & $N u_{x}$ \\
\hline 0.25 & -0.87596 & -0.33983 & 0.080647 & -2.58083 & 0.224274 & 0.25 & -0.87607 & -0.35701 & 0.08058 & -2.47789 & 0.235611 \\
\hline 0.5 & -0.8699 & -0.32419 & -0.39098 & -2.2554 & 0.213952 & 0.5 & -0.86999 & -0.34066 & -0.39108 & -2.15311 & 0.224823 \\
\hline 0.75 & -0.87314 & -0.30657 & -0.85342 & -1.95511 & 0.202327 & 0.75 & -0.87322 & -0.32218 & -0.85355 & -1.85238 & 0.212627 \\
\hline 1 & -0.88393 & -0.2865 & -1.31789 & -1.66675 & 0.189082 & 1 & -0.884 & -0.30105 & -1.31804 & -1.5627 & 0.19868 \\
\hline
\end{tabular}

Table V: Comparison of the missing values with $K$

\begin{tabular}{|c|c|c|c|c|c|c|c|c|c|c|}
\hline \multirow[t]{2}{*}{$\mathrm{K}$} & \multicolumn{2}{|c|}{$f^{\prime \prime}(0)$} & \multicolumn{2}{|c|}{$\theta^{\prime}(0)$} & \multicolumn{2}{|c|}{$h^{\prime}(0)$} & \multicolumn{2}{|r|}{$c_{f}$} & \multicolumn{2}{|c|}{$N u_{x}$} \\
\hline & Earlier & Present & Earlier & Present & Earlier & Present & Earlier & Present & Earlier & Present \\
\hline 0.4 & -0.39692 & -0.88631 & -0.55635 & -0.42843 & 2.419979 & -0.36831 & -0.6736 & -1.4077 & 0.3934 & 0.336604 \\
\hline 0.5 & -0.41335 & -0.87577 & -0.54396 & -0.43019 & 1.754125 & -0.37119 & -0.73071 & -1.45288 & 0.384636 & 0.337991 \\
\hline 0.6 & -0.41675 & -0.86539 & -0.54429 & -0.43192 & 1.499083 & -0.37108 & -0.76619 & -1.49686 & 0.384869 & 0.339349 \\
\hline 0.7 & -0.41666 & -0.85525 & -0.54592 & -0.4336 & 1.335808 & -0.36914 & -0.79548 & -1.5398 & 0.386022 & 0.340665 \\
\hline 0.8 & -0.4146 & -0.84541 & -0.54795 & -0.43521 & 1.216927 & -0.36604 & -0.82087 & -1.58185 & 0.387458 & 0.341934 \\
\hline 0.9 & -0.41138 & -0.83586 & -0.5501 & -0.43676 & 1.124516 & -0.3622 & -0.84358 & -1.6231 & 0.388982 & 0.343152 \\
\hline 1 & -0.40731 & -0.82662 & -0.55238 & -0.43825 & 1.049546 & -0.35789 & -0.86403 & -1.66361 & 0.39059 & 0.344318 \\
\hline
\end{tabular}

\section{CONCLUSION}

In this study, the effects of variable viscosity and thermal conductivity on the MHD flow of micropolar fluid on a continuous moving surface in the presence of a transverse magnetic field have studied considering the viscosity and thermal conductivity as the inverse linear functions of temperature where the microinertia density is assumed to be variable. The results demonstrate clearly that the viscosity and thermal conductivity parameters along with the other parameters such as $M, K, \theta_{r}, \theta_{c}$ and $N$ have significant effects on velocity, temperature, microinertia density and micro rotation distributions within the boundary layer. Thus assumption on constant properties may cause a significant error in flow problem.

\section{NOTATIONS}

$\mathrm{B}=$ magnetic intensity

$\mathrm{c}=$ dimensionless constant of integration

$\mathrm{c}_{\mathrm{p}}=$ Specific heat at constant pressure

$C_{\mathrm{f}}=$ Skin-friction coefficient

$E_{c}=$ Eckert number

$\mathrm{f}=$ Dimensionless stream function $\mathrm{g}=$ Dimensionless microinertia

$\mathrm{h}=$ Dimensionless angular velocity

$\mathrm{j}=$ Micro rotation density

$\mathrm{k}=$ Vortex viscosity

$\mathrm{K}=$ Coupling constant parameter

$M=\frac{2 \sigma B_{0}{ }^{2}}{\rho}$, Hartmann number

$\mathrm{n}=$ Constant

$\mathrm{N}=$ Angular velocity or component of the micro rotation vector normal to the xy plane

$\mathrm{Nu}_{\mathrm{x}}=$ Local Nusselt number

$P_{r}=\frac{\nu_{\infty} \rho \mathrm{c}_{\mathrm{p}}}{\lambda_{\infty}}$, Prandtl number

$q_{w}=$ Heat transfer from the plate

$\operatorname{Re}_{\mathrm{x}}=$ Local Reynolds number

$\mathrm{T}=$ Field temperature

$T_{w}=$ Plate temperature

$T_{\infty}=$ Ambient temperature 
$u=$ Velocity in the $x$-direction

$v=$ Velocity in the $y$-direction

$U_{w}=$ Plate velocity

$x, y=$ Cartesian coordinate along the surface and normal to it respectively

\section{Greek letters}

$\beta=$ Thermal expansion coefficient

$\gamma=$ Spin-gradient or micro rotation viscosity

$\kappa=$ Vortex viscosity

$\eta=$ Dimensionless co-ordinates

$\theta=$ Dimensionless temperature

$\theta_{c}=$ Dimensionless reference temperature corresponding to thermal conductivity parameter

$\theta_{r}=$ Dimensionless reference temperature corresponding to viscosity parameter

$v=$ Kinematic viscosity

$\mu=$ Dynamic viscosity

$\rho=$ Fluid density

$\tau_{w}=$ Skin friction

$\psi=$ Stream function

$\lambda=$ Thermal conductivity

\section{Subscripts:}

$\mathbf{w}$, the condition at the wall

$\infty$, the condition far away from the surface

Superscripts:

', Differentiation with respect to $\eta$

\section{REFERENCES}

[1] Abd El-hakiem M., Modather M., Abdou M, The effect of variable viscosity on MHD natural convection in micropolar fluids, Int. J. Appl. Mech. Engg., 11, 2( 2006), pp. 301-320.

[2] Eringen A.C., Simple micro fluids, Int. J. Eng. Sci., 2 (1964) pp. 205-217

[3] Eringen A.C., Theory of micropolar fluids, J. Math. Mech., 16, (1966), pp.1-18

[4] Eringen A.C., Theory of micropolar fluids, J. Math. Anal. Appl., 38(1972), pp.480- 496

[5] Gorla R.S., Heat transfer in micropolar boundary layer flow over a flat plate, Int. J. Eng. Sci., 21 (1983), pp. 791-796

[6] Gorla R.S.R., Combined forced and free convection in micropolar boundary layer flow on a vertical flat plate, Int. J. Engng. Sci., 26, 385-391, 1988.

[7] Gorla R.S.R., Takhar H.S., Slaouti A., Magneto hydrodynamic free convection boundary layer flow of a thermo micropolar fluid over a vertical plate, Int. J. Eng. Sci., 36(1998) pp. 315-327
[8] Guram G.S., Smith A.C., Stagnation flow of micropolar fluids with strong and weak interactions, Comp. Math. With Appl., 6, 213-233, 1980

[9] Hoyt J.W., Fabula A.G., The effect of additives on fluid friction, U S Naval Ordinance Test Station Report, 1964

[10] Ishak A., Nazar R., Pop I., Flow of a micropolar fluid on a continuous moving surface, Arch. Mech., 58,6,(2006), pp.529-541

[11] Khound P.K., Hazarika G.C., The effect of variable viscosity and thermal conductivity on liquid film on an unsteady stretching surface, Proc. of $46^{\text {th }}$ Annual Tech. Session, Ass Sc. Soc. 2000, pp. 47-56

[12] Lai, F.C. and Kulacki, F.A., The effect of variable viscosity on convective heat and mass transfer along a vertical surface in saturated porous medium, Int. J.Heat and Mass Transfer 33, 1028-1031

[13] Muthucumaraswami R., Sundar M., Subramonium V.S.A., Magnetic field effects on flow past an accelerated isothermal vertical plate with heat and mass diffusion, Annals of faculty Engineering, Hunedoara- Int. J. of Eng., 2012,pp.177-180

[14] Modather M., Abdou M., Roshdy El-Zahar E., Variable viscosity effect on heat transfer over a continuous moving surface with variable internal heat generation in micropolar fluids, Appl.Math. Sci, 6,128, 2012, pp. 6365-6379

[15] Peddieson J., Mecnitt R. P., Boundary layer theory for a micropolar fluid, Recent Adv. Engng. Sci., 5 (1997) pp. $405-425$

[16] Rajesh V., MHD free convection flow past an accelerated vertical porous plate with variable temperature through a porous medium, Acta Technica Corviniensis, Bulletin and Engineering, pp.91-96

[17] 17. Salem A. M., Odda S. N., Influence of variable viscosity and thermal conductivity on flow of micropolar fluid past a continuously moving plate with suction or injection, The Korean society for Ind. Appl. Math., 2005

[18] Sarma U., Hazarika G. C., Effects of variable viscosity and thermal conductivity on heat and mass transfer flow along a vertical plate in the presence magnetic field, Int. J. Phy. Education,

[19] Sarma U., Hazarika G. C. Effects of variable viscosity and thermal conductivity on combined free-forced convection and mass transfer flow passed a vertical porous plate, Antarctica J. Math., 7(4), 2010, pp.461-472

[20] Soundalgekar V.M., Takhar H.S., Flow of micropolar fluid past a continuously moving plate with vriable spin gradient viscosity, Acta Mech., 131, 139-151, 1998

[21] Vogel W.M., Patterson A.M., An experimental investigation of additives injected into the boundary layer of an underwater body, Pacific Naval Lab. of the Defence Res. Board of Canada, Report 64-2, 1964. 